\title{
Deployable Structures in Plants
}

\author{
Hidetoshi Kobayashi ${ }^{1, a}$ and Keitaro Horikawa ${ }^{2, b}$ \\ ${ }^{1,2}$ Department of Mechanical Science and Bioengineering, \\ Graduate School of Engineering Science, Osaka University, \\ 1-3, Machikaneyama, Toyonaka, 560-8531, Japan

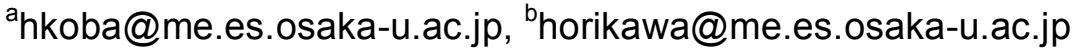

Keywords: Corrugated leaf, Vector analysis, Vein angle, Sympetalous flower, Petal number

\begin{abstract}
The deployment of leaves with plane surface and straight parallel folds, as observed in leaves of hornbeam and beech, was investigated by using numerical methods. In both species the veins are angled at $30^{\circ}$ to $50^{\circ}$ from the midrib, when the leaves are outstretched. Although a higher angle allows the leaf to be folded more compactly within the bud, it has very small leaf area in the early stage of unfolding. The midrib of leaf grows very slowly at first and then it does with an almost constant speed. From the numerical simulation, it was found that the midrib grows with the minimum unfolding energy. The deployment of flowers was also investigated from mechanical point of view. A potato flower has five or six petals with triangle gussets between petals. The bud volume becomes largest when the number of petals, $N$, is five. However, the energy for unfolding of the model with $N=5$ or 6 is smaller than those of other models, if the energy can be represented by the total kinetic energy during unfolding.
\end{abstract}

\section{Introduction}

With the coming of spring, many leaves and flowers come out of the buds and grow to their normal size. Most leaves or flowers not only grow but also deploy or unfold from their small buds. In the tiny buds of leaves or flowers, therefore, various ideas to stow their bodies such as folding or rolling are often observed. For example, The leaves of hornbeam or common beech have a typical corrugated folding pattern [1,2] while the fan-type bellows pattern is observed in maple leaves [3]. As well-known, the petals or petal ribs of a morning glory flower mysteriously spiral in a bud [4] and there are foldable triangle gussets between individual petals in potato flowers. With respect to the opening or closing of flowers, relatively many researches have been performed from the point of plant physiology. Photonarsty and thermonasty observed in the opening of morning glory [5] and oxalis [6] flowers were investigated experimentally. Two models were developed and compared for predicting the unfolding of chrysanthemum leaves in relation to mean day temperature and daily light integral [7] and it was concluded that the most influential on the rapid opening of lily flowers is the change in angle between the midrib of petals and the pedicel [8]. A number of deployable structures observed in nature are summarized and classified by Kresling [9]. The investigation of the folding or unfolding manner in plants [10], therefore, may be useful in designing some artificial deployable structures [11-13], such as solar panels, antenna of satellites, or deployable roofs. Although the variation in leaflet angles of sensitive plant and compaction after folding were examined by using leaf models with experimental data [14], there are generally little researches concerning folding or unfolding manner in plants from mechanical point of view.

In this paper, the folding or unfolding manner of leaves with corrugated folding pattern like hornbeam or common beech leaves and potato flowers were are investigated from mechanical view point as examples for deployable structure in plants by using observation and numerical simulation.

\section{Unfolding of Corrugated Leaves}

Vein Angle of Leaves Unfolded. Unfolding leaves of hornbeam (Carpinus betulus) are shown in Fig. 1. The leaves develop within a bud where they are protected by several layers of bud scales. In 
spring the folded leaves emerge, revealing their regular folding pattern. Since the folds or creases in these leaves are along veins, the vein angle, $\alpha$, and the location of the veins, $X$, were measured. Here, $\alpha$ is defined as the angle between the midrib and the vein and $X$ is measured from the boundary between the petiole and the leaf lamella. Fig. 2 shows the relation between $\alpha$ and $X$ of hornbeam and common beech (Fagus sylvaticus) leaves obtained from two leaves individually. A non-dimensional parameter $X^{*}\left(=X / L_{0}\right.$ where $L_{0}$ is the length of the lamina) is used to characterize the position of the veins. The vein angles in hornbeam and beech are not constant but decrease gradually from about $50^{\circ}$ to $30^{\circ}$ toward the tip of the leaf. The average vein angle is about $40^{\circ}$ in both leaves. The veins transport water and assimilation products within the leaf as well as providing mechanical support [15], so their geometry may be related to either of these functions.

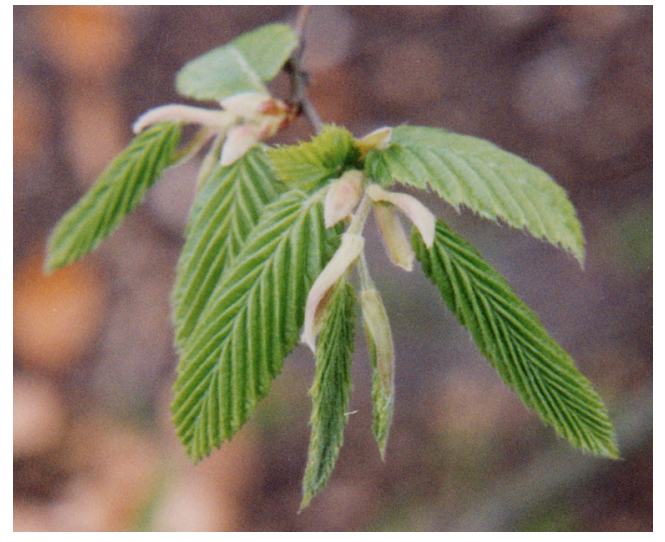

Fig. 1 Unfolding of hornbeam corrugated leaves.

Fig. 2 Vein angles measured in leaves of (a) hornbeam and (b) common beech.
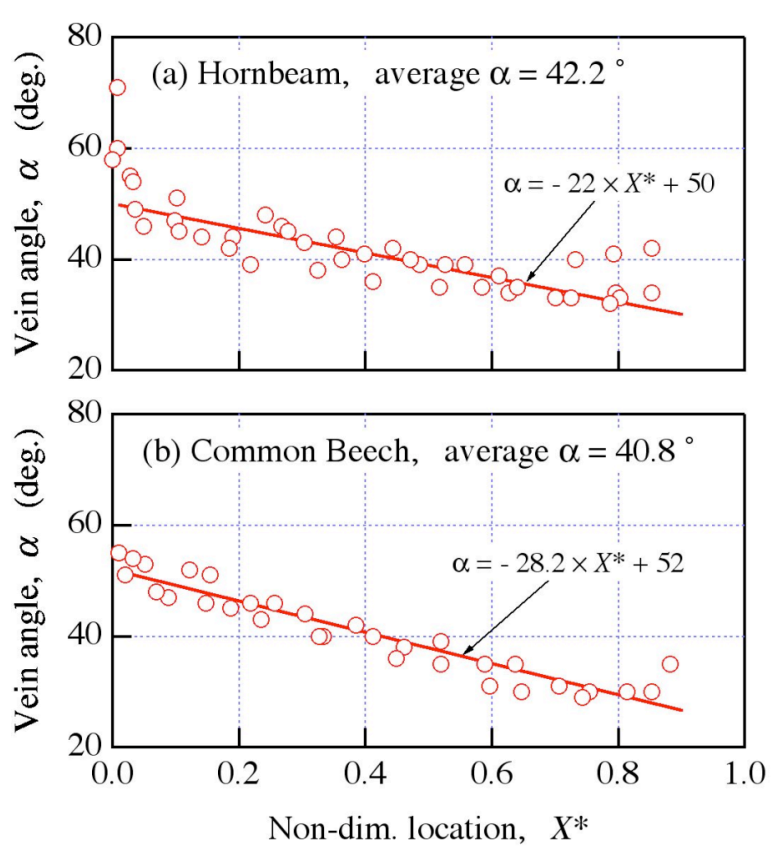

Paper Models of Corrugated Leaf. In order to simulate the unfolding of these corrugated leaves, a type of paper leaf model including crest and valley creases was considered, as shown in Fig. 3(a). This folding pattern is a simple form of the folding pattern known as "Miura-Ori" [12]. A sheet folded in the "Miura-Ori" pattern can extend not only in the pulling direction but also in the direction perpendicular to the pulling direction. Since this type of leaf model has symmetric folding and unfolding with respect to the midrib, we considered only half of the leaf. For simplicity, the distance between creases and the inclined angle of the creases (corresponding to vein angles, $\alpha$ ) are constant along the length of the leaf. It is also assumed that the lamina elements divided by the creases were stiff enough; therefore, the deformation of the lamina elements during unfolding was not taken into account. Furthermore, the thickness of the model leaf was considered to be negligible compared with the leaf size.

The side views of the leaf paper models with different $\alpha,\left(30^{\circ}, 45^{\circ}, 60^{\circ}, 75^{\circ}, 85^{\circ}\right)$ fully folded are shown in Fig. 3(b). The portions shaded indicate the reverse surface. The shape and the area of side views, thus, depend on the vein angle, $\alpha$. Leaves with larger $\alpha$ can be folded more compactly.

The area ratios, $A_{F}{ }^{*}$ which is the ratio between $A_{F}$ (lateral area of model leaves) and $A_{0}$ (unfolded leaf area), i.e. $A_{F}{ }^{*}=A_{F} / A_{0}$, are shown in Fig. 3(c). In the models, the leaf with $\alpha=45^{\circ}$ as the largest $A_{F}{ }^{*}$. The $A_{F}{ }^{*}$ of the leaf with $\alpha=85^{\circ}$ is about half of that with $\alpha=45^{\circ}$. By folding with 8 creases, the surface area of the leaf can be reduced to $10-20 \%$ of an unfolded leaf, although the reduction depends on the number of creases, of course. A small $A_{F}{ }^{*}$ is probably more suitable for storing folded leaves into a small space. Therefore, the leaves with large $\alpha\left(75^{\circ}\right.$ or $\left.85^{\circ}\right)$, have an advantage for compact housing in the bud. 

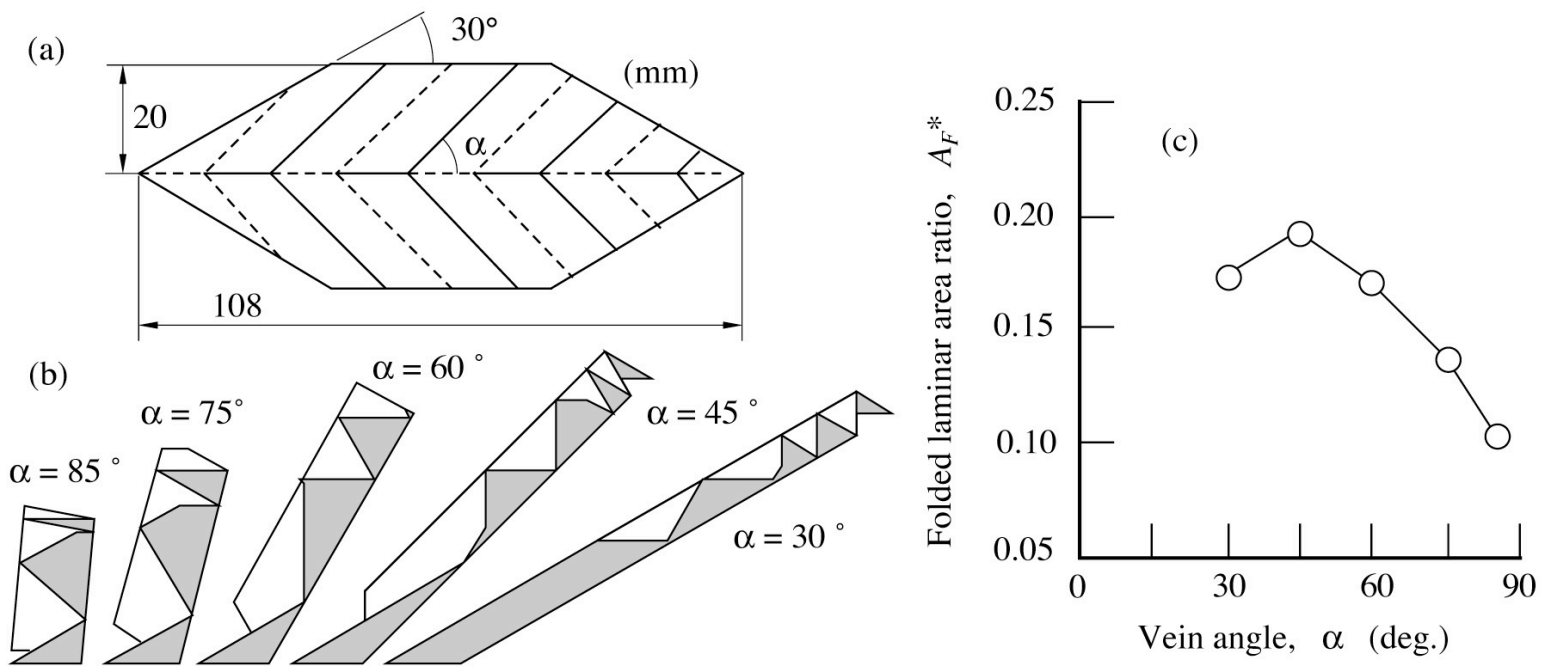

Fig. 3 Leaf paper models: (a) shape of unfolded leaf model with $\alpha=45^{\circ}$, (b) side views of fully folded leaf models and (c) folded leaf area ratio of leaf models with various $\alpha$.

Numerical Simulation for Unfolding of Leaf Models. In order to determine the location of creases in a three-dimensional space, vector analysis was carried out using $\mathrm{O}-x y z$ coordinates, as shown in Fig.4. The $x z$-plane is a plane of symmetry and the origin, $\mathrm{O}$, is located at the boundary point between the petiole and the midrib. The opening angle, $\theta$, is defined as the angle between the plane of symmetry and the nearest lamina element to the origin, O. Before the leaf starts unfolding, $\theta=0^{\circ}$. When the leaf is fully unfolded, $\theta=90^{\circ}$. The axis of $x^{\prime}$ indicates a direction linking the crests of the zigzag midrib during unfolding, which is the direction perpendicular to the $y$ axis on the $x z$-plane. The angle between $x$ and $x^{\prime}$ axes changes during unfolding. A simple structure included in the region $\mathrm{R}$ consists of four lamina elements and four creases, as shown in Fig. 4(b). This structure is repeated throughout the entire leaf. Therefore, once two unit vectors, $\boldsymbol{p}$ and $\boldsymbol{q}$, on the creases are expressed by $\theta$ and $\alpha$ given, it can be generalized easily to the calculation of the entire leaf unfolding by the transformation of coordinates. The unit vector $\boldsymbol{p}$ can be obtained easily from the geometry shown in Fig. 4(c) as follows :

$$
\boldsymbol{p}=\cos \alpha \boldsymbol{i}+\sin \alpha \sin \theta \boldsymbol{j}+\sin \alpha \cos \theta \boldsymbol{k}, \quad \boldsymbol{q}=q_{x} \boldsymbol{i}+q_{z} \boldsymbol{k} .
$$
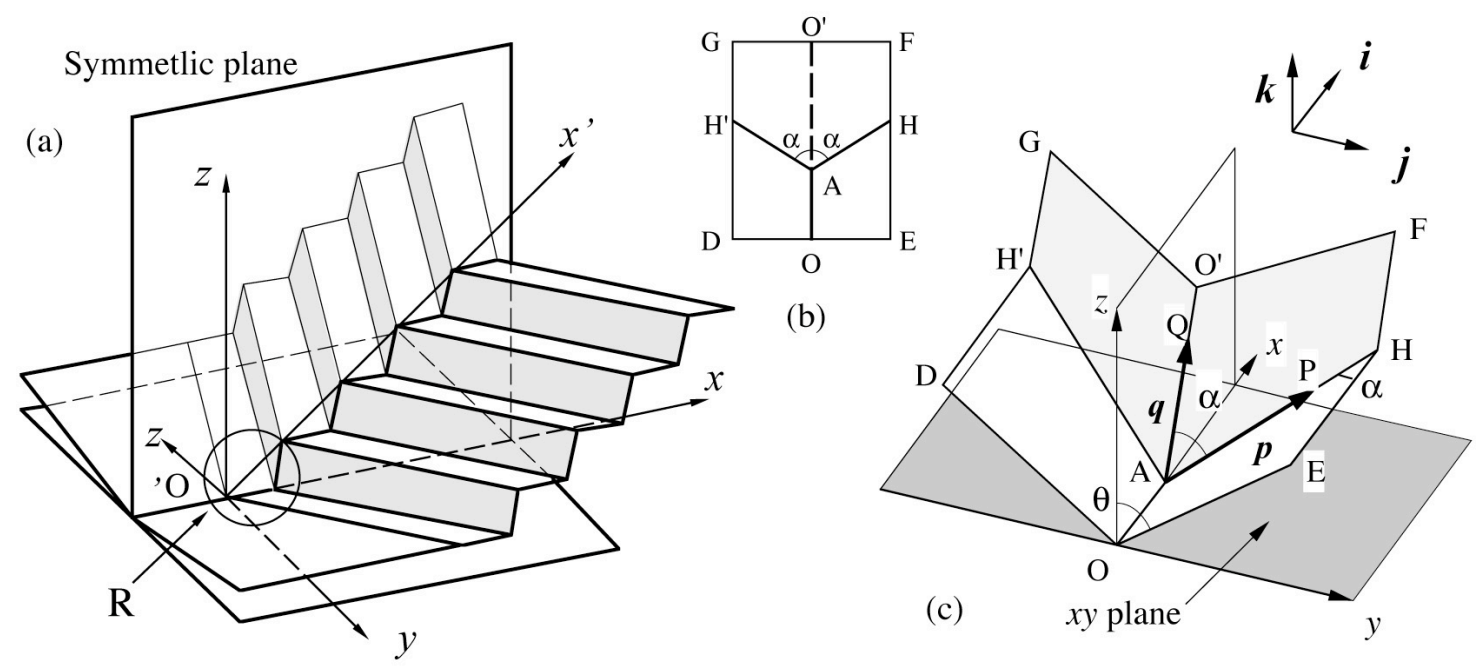

Fig. 4 Corrugated model (simple form of Miura-Ori) and coordinates used for vector analysis. 
where $\boldsymbol{i}, \boldsymbol{j}$ and $\boldsymbol{k}$ are the unit vectors in the $x, y$ and $z$ directions, respectively. And $q_{x}$ and $q_{z}$ are unknown components of vector $\boldsymbol{q}$ in the $x$ and $z$ directions, respectively. Since the angle between $\boldsymbol{p}$ and $\boldsymbol{q}$ is $\alpha$ and both their lengths are 1, the scalar product of $\boldsymbol{p}$ and $\boldsymbol{q}$ can be shown by

$$
\boldsymbol{p} \bullet \boldsymbol{q}=q_{x} \cos \alpha+q_{z} \sin \alpha \cos \theta=|\boldsymbol{p} \| \boldsymbol{q}| \cos \alpha=\cos \alpha, \quad q_{x}^{2}+q_{z}^{2}=1
$$

Solving Eq. 2 for $q_{x}$ and $q_{z}$, the following equations can be obtained.

$$
q_{x}=\frac{1-h^{2}}{1+h^{2}} \quad, \quad q_{z}=\frac{2 h}{1+h^{2}} \quad, \quad h=\tan \alpha \cos \theta
$$

Eqs. 1 and 3 give the directions of creases in the region $\mathrm{R}$ when $\alpha$ and $\theta$ are known. By repetition of this procedure, the locations of all creases and intersection points in three dimensional space can be determined. More details concerning the simulation are given in the previous study [1].

Leaf Area during Unfolding. To examine the behaviour of unfolding leaves, the area of leaf models projected on to the $x^{\prime} y$-plane (see Fig. 4), $A$, was calculated at several opening angles, $\theta$. Fig. 5 shows the change of projected leaf area ratio, $A^{*}\left(=A / A_{0}\right)$, of all leaf models during unfolding. The leaf model with $\alpha=30^{\circ}$ already has $60 \%$ of the fully unfolded area, $A_{0}$, at $\theta=40^{\circ}$. However, at the same value of $\theta$, the model with $\alpha=85^{\circ}$ has less than $10 \%$ of $A_{0}$. In order to photosynthesize effectively, an amount of leaf area expanded is needed. For all plants, therefore, timing is presumably important only where the habitat has annual cycles. Away from tropical areas where there is a limited growing season, one might expect the leaf to be deployed as quickly as possible, once the temperature and light intensity become favorable. Thus, if all leaf models take the same timing at 50\% and $100 \%$ deployments, the curves shown in Fig. 5 can be redrawn like Fig. 6 . The horizontal axis shows non-dimensional time, $T$, in which the total unfolding time of the leaf model with $\alpha=45^{\circ}$ equals to one. It is clearly found that the leaf with large vein angle needs early deployment. This early deployment is very dangerous for leaves, because this will expose the leaf to damage by late frosts and to attract herbivores early in the season when green food is scarce. The leaves with vein angle of $30^{\circ} \sim 60^{\circ}$ might be at an advantage in defending itself from this sort of damage and can deploy more quickly, although they cannot fold compactly.

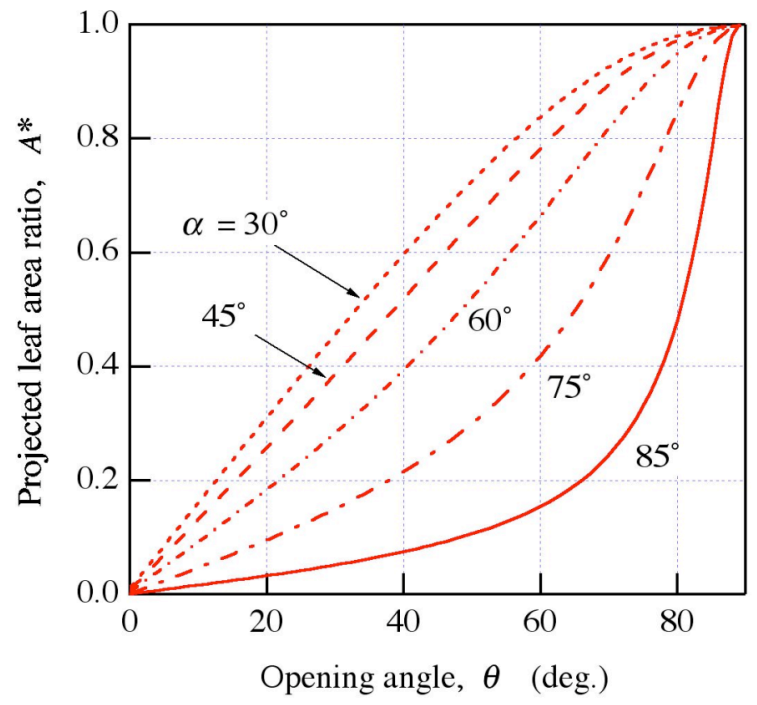

Fig. 5 Change of projected leaf area of all leaf models during unfolding.

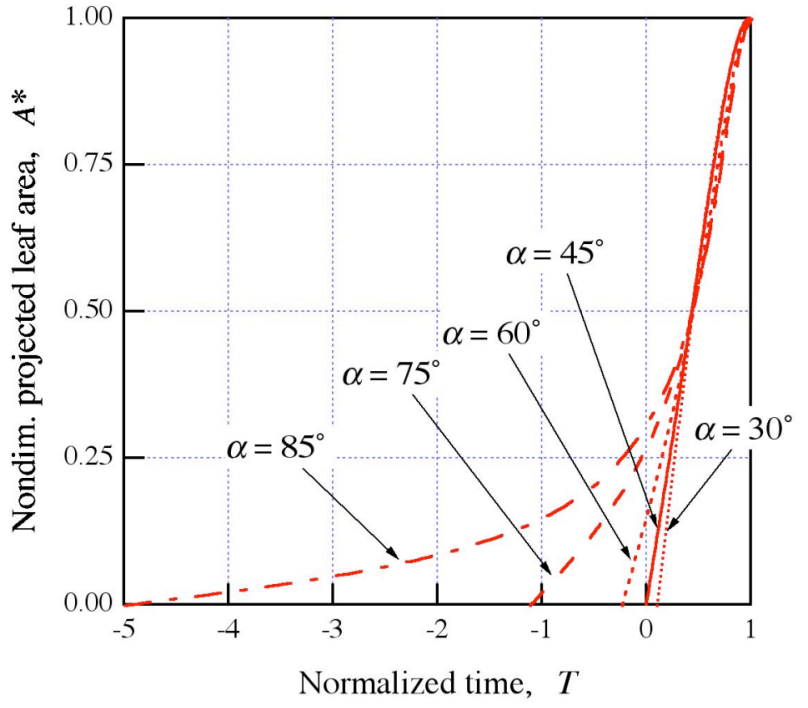

Fig. 6 Change of projected leaf area when timing at $50 \%$ to $100 \%$ opening is all the same. 
Midrib Growth and Kinetic Energy during Unfolding. Unfolding of corrugated leaves is usually caused by the growth of midrib. The growth of midrib is accompanied by the growth of cells in the midrib due to water absorption which is caused by osmotic pressure in cells. Since energy is consumed to maintain the osmotic pressure [16], the cell growth due to water absorption may be in proportion to the energy consumed in plants, i.e. the change of midrib length is relevant to the energy consumed during leaf unfolding. Fig. 7 shows the growth of midrib of two Monarch birch leaves, which have also corrugated folding pattern. From the data of leaf- $\mathrm{A}$ in this figure, it is found that very slow initial growth of midrib is accompanied by almost constant growth after.

The amount of kinetic energy of leaf during unfolding is so small because the leaves open so slowly. However, the changing manner of the kinetic energy is relevant or my be in proportion to the midrib growth, because the unfolding of corrugated leaves is caused by the midrib growth. This means that the kinetic energy may represent the energy consumed in the unfolding of corrugated leaves. To estimate the kinetic energy, a number of additional assumptions were used, i.e., the uniform thickness of leaf, $t$, and a homogeneous leaf material, whose density is $\rho$, for simplicity. If the mass of a lamina element can be concentrated to the centroid of the elements, the movement of particles can be used for the calculation of kinetic energy instead of considering the movement of the lamina elements. The kinetic energy of a leaf model, $w$, is given by

$$
w=\sum_{i=1}^{n} \frac{1}{2}\left(\rho t A_{i}\right) V_{i}^{2}
$$

where, $A_{i}$ and $V_{i}$ are the area and the velocity of the $i$-th element and $n$ is the total number of elements. Using Eq. 4, the change of the kinetic energy during unfolding [1] and the total energy for fully unfolding, $W$, can be calculated. When the average kinetic energy during a small time interval, $\Delta T$, is denoted by $w_{j}$ (the suffix $j$ is time step), the $W$ is given by $W=\Sigma\left(w_{j} \Delta T\right)$. Since $w_{j}$ is positive and independent each other, a relation is derived from arithmetic and geometric average, as follows:

$$
W=\Delta T \cdot \sum_{j=1}^{m} w_{j} \geq \Delta T \cdot m \cdot\left(\prod_{j=1}^{m} w_{j}\right)^{\frac{1}{m}}
$$

where $m$ is the number of total time steps. The equality in Eq. 5 is valid when $w_{1}=w_{2}=\ldots=w_{m}$. Therefore, the total energy becomes a minimum when the kinetic energy $w$ is kept constant during unfolding. Fig. 8 shows the change of midrib length during unfolding under the minimum energy

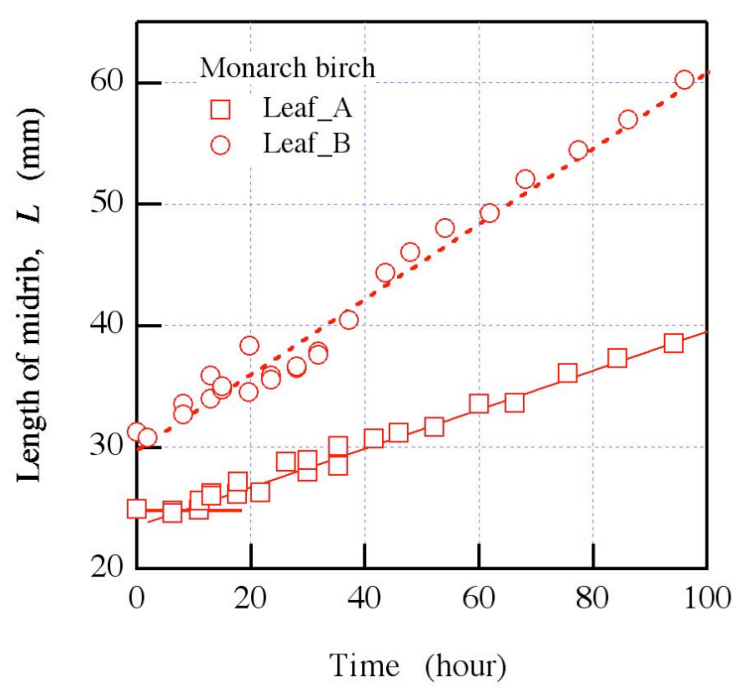

Fig. 7 Midrib growth of Monarch birch leaves.

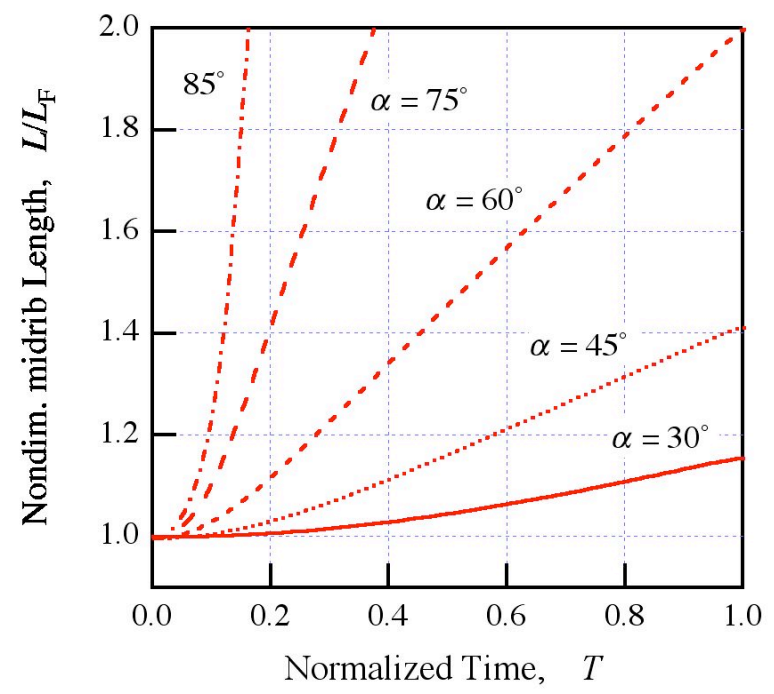

Fig. 8 Extension of midrib under minimum energy condition. 
condition. The curve of the leaf model with $\alpha=45^{\circ}$ is quite similar to the growth curve of the midrib measured, shown in Fig. 7, i.e. the growth is very slow at first and almost constant after that. This means that the midrib of birch leaves grows with a minimum of energy, if the kinetic energy of corrugated leaves during unfolding represents the energy required for the deployment of leaves.

\section{Unfolding of Potato Flower}

Observation of Potato Flower. A potato flower has usually five or six petals and foldable gussets are between individual petals, as shown in Fig. 9(a). After unfolding fully, the petals and gussets make a plane like a pentagon or hexagon. Therefore, we can refer to the folding manner of potato flowers in the consideration for the folding manner of polygonal planes. Since the back of only petals can be seen in a bud, the gusset is folded and inserted in the bud. In order to satisfy this situation, we introduced a number of creases into the gussets. In a real bud of potato flowers, the gusset is folded in two and curved or rolled, because of a thin membrane. However, we adopt a number of foldable rigid thin plates instead of the membrane as the gussets here, for simplicity. A plane made by petals and gussets fully unfolded was modeled by a regular polygon with the number of side, $N$, (corresponding to petal number) and area, $A_{0}$. As shown in Fig. 9 (b), it can be divided into $2 N$ unit right triangles. A unit right triangle $\triangle \mathrm{AOD}\left(\angle \mathrm{ADO}=90^{\circ}\right)$ consists of a half petal (while area) and a half gusset (shadow area) with two creases, a valley crease OB (dashed line) and a crest crease OC (solid line), as shown in Fig 9(c). These creases divide $\triangle$ AOD into three partial triangles, therefore, the central angle, $\alpha(=\angle \mathrm{AOD}=\pi / N)$, is also divided into three partial angles, $\beta_{1} \sim \beta_{3}$. From the symmetry of a regular polygon, we just consider the folding or unfolding of the unit right triangle after this.
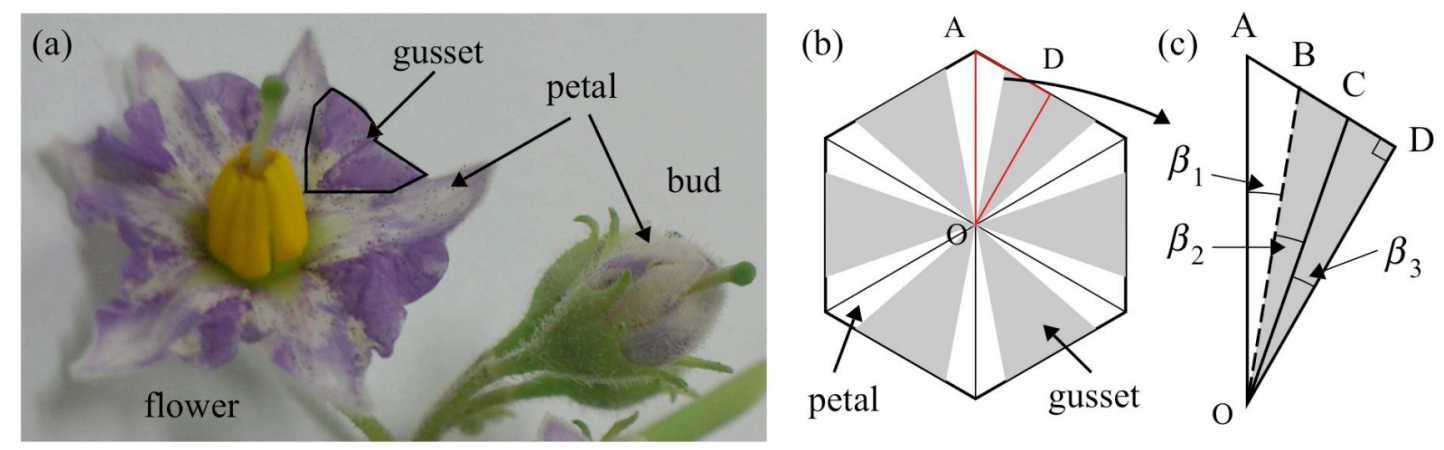

Fig. 9 Potato flower: (a) unfolded flower and bud, (b) hexagonal model and (c) unit right triangle.

Coordinate System and Assumptions for Numerical Simulation. For the numerical simulation of flower unfolding, O-xyz coordinates were adopted as shown in Fig. 10. The plane I ( $x z$-plane) and plane II, crossing each other at $z$-axis with $\alpha$, are the planes of symmetry. The origin, $\mathrm{O}$, is the centre of the polygon models, which are six regular polygons, triangle, square, pentagon, hexagon, octagon and dodecagon (i.e. $N=3,4,5,6,8$ and 12). When the polygon is fully unfolded, the unit triangle is on $x y$-plane, as shown in Fig. 10. By folding along the creases, its body changes to a zigzag slender body with three partial triangles, $\triangle \mathrm{AOB}, \triangle \mathrm{BOC}, \triangle \mathrm{COD}$. The opening angle, $\theta$, is defined as the angle between $z$-axis and the line OA. Before the petal starts unfolding, $\theta=\theta_{0}$. When the petal is fully unfolded, $\theta=90^{\circ}$. The angles $\phi$ and $\psi$ are also defined as the angles between $z$-axis and the lines $\mathrm{OB}$ and $\mathrm{OD}$, respectively. To calculate the location of points $\mathrm{A} \sim \mathrm{D}$ during unfolding, we made a number of assumptions and limitations in the simulation as follows:

1. Folding and unfolding are symmetrical about the plane I and II.

2. Even after the unit triangle is folded, its body must be in the space between planes I and II. This 
means that the lines OA and OD move on the plane I and II, respectively, during unfolding.

3. When the unit triangle is fully folded, the line OB is on the plane II.

4. $\triangle \mathrm{AOB}$ and $\triangle \mathrm{COD}$ are perpendicular to plane I and plane II, respectively.

5. Three partial triangles, $\triangle \mathrm{AOB}, \triangle \mathrm{BOC}, \triangle \mathrm{COD}$ have the flat rigid bodies so that their deformation during unfolding can be neglected. Thus, the area of outstretched surface, $\triangle \mathrm{A}^{\prime} \mathrm{OD}^{\prime}$ is identical to the folded one.
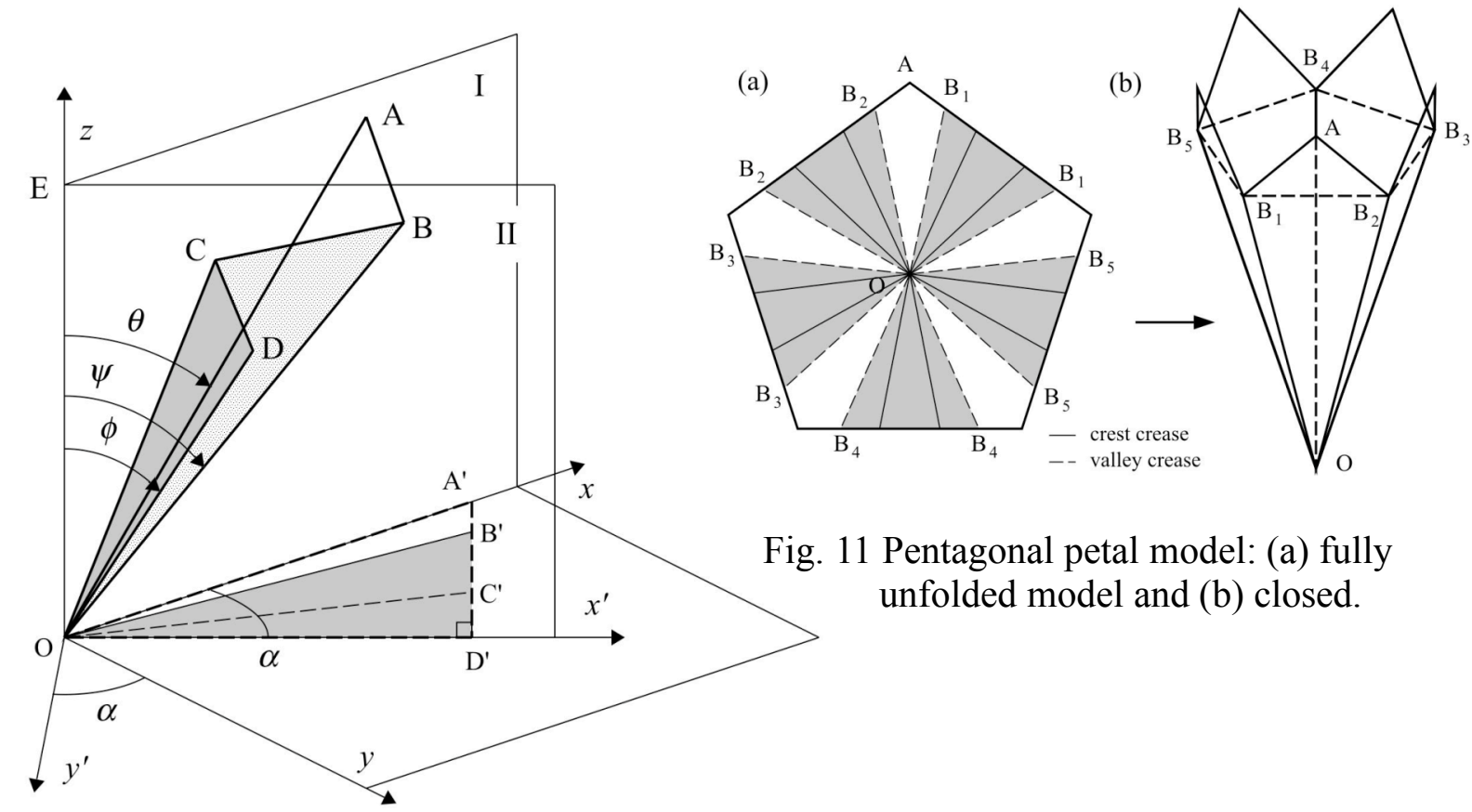

Fig. 11 Pentagonal petal model: (a) fully unfolded model and (b) closed.

Fig. 10 Unit right triangle and coordinates used for simulation.

Bud Volume and Partial Divding Angle. Fig. 11 shows a regular pentagon with the area $A_{0}$ as an example of the polygonal models. When it is fully folded along the creases, it makes a pentagonal pyramid $A-B_{1} B_{2} B_{3} B_{4} B_{5}$ shown in Fig. 11(b). We define the bud volume as the volume of the pentagonal pyramid here, although the tip triangles like $\triangle \mathrm{AB}_{1} \mathrm{~B}_{2}$ are left. In order to determine three partial dividing angles, $\beta_{1} \sim \beta_{3}$ in the unit right triangle $\triangle \mathrm{AOD}$ (see Fig. 9), the minimum bud volume condition is used. From the geometrical relations in the model fully folded, the bud volume $V$ can be expressed by using the circumradius $R$ of the pentagon and the initial opening angle $\theta_{0}$ as follows:

$$
V=\frac{N R^{3} \tan \alpha}{3} \cdot \frac{\sin ^{2} \theta_{0} \cos \theta_{0}}{\left(\tan ^{2} \alpha \sin \theta_{0}+1\right)^{3}} \quad, \quad R=\sqrt{\frac{2 A_{0}}{N \sin 2 \alpha}}
$$

If $\sin \theta<\left(-\tan ^{2} \alpha+\sqrt{\tan ^{4} \alpha+24}\right) / 6$, the volume $V$ simply increases with the increase in $\theta$. The most severe condition appears at $N=3$, which is $\sin \theta<0.46$. This means that it is enough to choose the smallest $\theta_{0}$ to obtain the minimum bud volume. From the assumptions and the geometrical relations shown above, the following equations can be obtained:

$$
\begin{aligned}
& \tan \beta_{1}=\tan \alpha \sin \theta_{0}, \quad \tan \beta_{3}=\tan \alpha \sin \phi_{0}, \quad \beta_{1}+\beta_{2}+\beta_{3}=\alpha \\
& \tan \theta_{\mathrm{C}}=\frac{\tan \phi_{0}}{\cos \alpha}, \quad \frac{\cos \beta_{2}}{\cos \beta_{1}}=\cos \theta_{0} \cos \theta_{\mathrm{C}}\left(\tan \theta_{0} \tan \theta_{\mathrm{C}}+1\right)
\end{aligned}
$$


where $\phi_{0}$ is the initial value of $\phi$ and $\theta_{\mathrm{C}}$ is $\angle \mathrm{COE}$. There are five equations and six unknowns, $\beta_{1}$, $\beta_{2}, \beta_{3}, \theta_{0}, \phi_{0}$ and $\theta_{\mathrm{C}}$. Although the unknowns cannot be determined easily, a relation between $\beta_{3}$ and $\theta_{\mathrm{C}}$ can be derived as follows:

$$
\begin{aligned}
& \left(B^{2}-A^{2}\right) \tan ^{2} \theta_{0}+2 B C \tan \theta_{0}+C^{2}-A^{2}=0 \\
& A=1+\tan \alpha \tan \beta_{3}, \quad B=\frac{\tan \beta_{3}\left(1+2 \tan ^{2} \alpha\right)-\tan ^{3} \alpha}{\tan \alpha}, C=\frac{\sqrt{\left(1+\tan ^{2} \alpha\right)\left(\tan ^{2} \alpha-\tan ^{2} \beta_{3}\right)}}{\tan \alpha}
\end{aligned}
$$

Since the first equation in Eq. 8 is a quadratic equation with respect to $\tan \theta_{0}$, we can solve it easily by using the quadratic formula. Fig. 12 shows the change of five angles with $\beta_{3}$ in the case of $N=5$. $\beta_{2}$ must be larger than $\beta_{1}$, because $\triangle \mathrm{BOC}$ is more inside than $\triangle \mathrm{AOB}$, as seen in Fig. 10 . In the region up to the limit of $\beta_{3}=10.53^{\circ}$, the curve of $\theta_{0}$ becomes convex upward. The minimum value of $\theta_{0}$ appears on the limit line and $\theta_{0}=18.12^{\circ}$. From this, we can obtain $\beta_{1}=\beta_{2}=12.73^{\circ}$. Fig. 13 shows the change of $\theta_{0}$ of all models with various $N$ up to the individual limit lines. Since all curves are basically convex upward, the point at which $\theta_{0}$ becomes the minimum appears on the limit line or on the line of $\beta_{3}=0$. When $N \leq 5$, the minimum $\theta_{0}$ appears on the limit line, i.e. $\beta_{1}=\beta_{2}$. When $N$ $\geq 6$, i.e. $\beta_{3}=0$, the unit triangle $\triangle$ AOD is divided into two partial triangles. This means that dual portioning is more suitable than division into three when $N \geq 6$. For the vector analysis shown below, the numerical values of $\beta_{1} \sim \beta_{3}$ determined here were used.

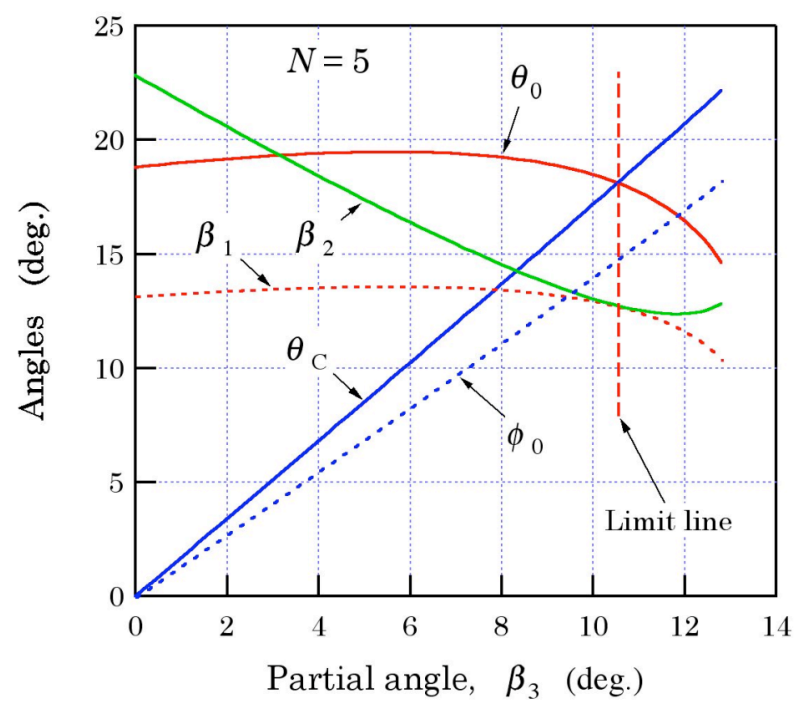

Fig. 12 Change of various angles with $\beta_{3}(N=5)$.

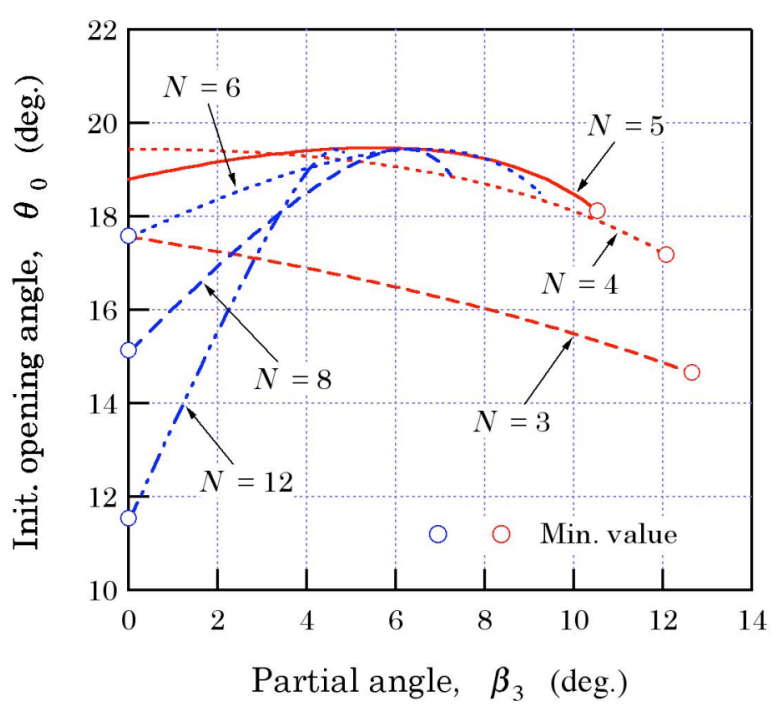

Fig. 13 Change of $\theta_{0}$ of six different polygonal models.

Kinetic Energy during Unfolding, By using vector analysis, we can obtain the location of the partial triangles, $\triangle \mathrm{AOB}, \triangle \mathrm{BOC}$ and $\triangle \mathrm{COD}$. These triangles revolve about the axes on $x y$-plane during unfolding. Thus, if we use the suitable moment of inertia of the partial triangles $\left(I_{1}, I_{2}\right.$ and $I_{3}$, respectively), their kinetic energies can be calculated. As mentioned above, the amount of kinetic energy of petals and gussets is not so large. However, if it can be considered that the changing manner of kinetic energy is relevant to that of the energy consumed for flower unfolding as well as leaf unfolding, to examine the kinetic energy of models may be meaningful. Since there is not enough space here, the details of the calculation are omitted. Fig. 14 shows how the kinetic energies of $\triangle \mathrm{BOC}$ and $\triangle \mathrm{COD}$ change when $\triangle \mathrm{AOB}$ is unfolded with a constant angular velocity, $\omega$. The 
horizontal axis is the non-dimensional time $T$, i.e. $T=0$ and 1 mean fully folded and unfolded, respectively. The petal model used here is a pentagonal model, i.e. $N=5$. Since $\triangle \mathrm{BOC}$ connects to $\triangle \mathrm{AOB}$ through a crease $\mathrm{OB}$, the energy of $\triangle \mathrm{BOC}\left(\mathrm{I}_{2} \omega^{2}\right.$ in Fig. 14) changes similarly to that of $\triangle \mathrm{AOB}$, i.e. almost constant. However, $I_{3} \omega^{2}$, which is the energy of $\triangle \mathrm{COD}$, gradually increases because the effect of the movement of $\triangle \mathrm{AOB}$ is reduced by the rotation of $\triangle \mathrm{BOC}$ around $\mathrm{OB}$. The area under the curves gives the kinetic energy used for fully unfolding. By adding up the kinetic energy of individual triangles, the total energy of a model for fully unfolding can be calculated. Similar calculation was carried out for other models keeping the total unfolding time constant. Fig. 15 shows the total energy ratio $W^{*}$ to the energy of the model with $N=5$. The bud volume ratio $V^{*}$ calculated by Eq. 6 is also shown. From this figure, the total energy of the model with $N=5$ or 6 is smaller than that of other models. On the contrary, the bud volume is larger. Potato flower has usually 5 or 6 petals, so it can be said that potato adopts the most effective petal number for unfolding kinetic energy, although the bud is not compact.

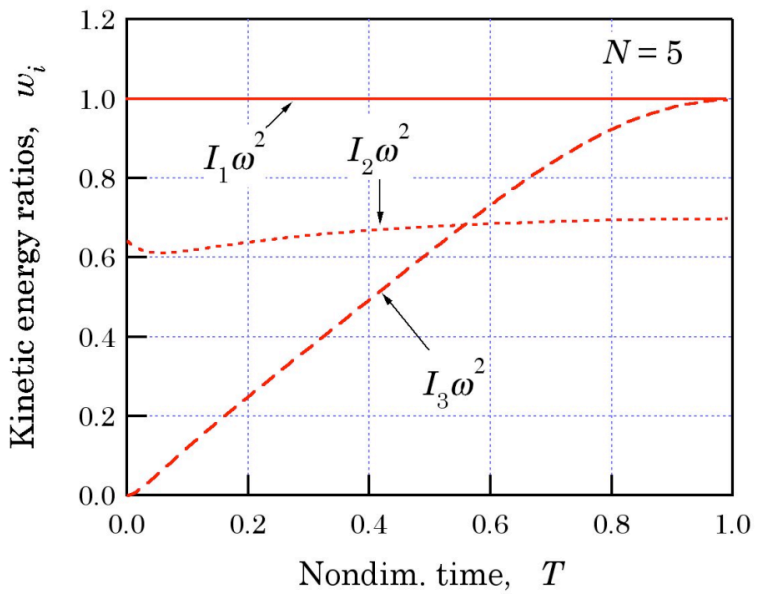

Fig. 14 Kinetic energy of partial triangles during unfolding $(N=5)$.

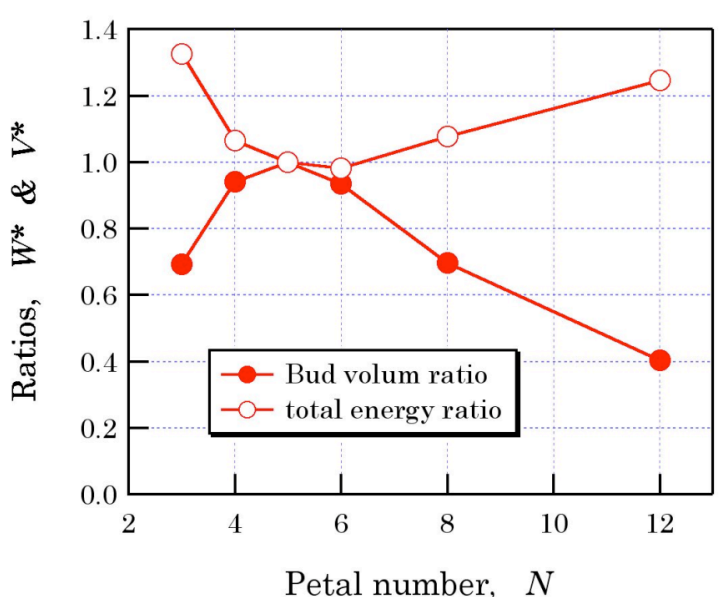

Fig. 15 Total kinetic energy ratio and bud volume ratio.

\section{Summary}

The deployment of leaves with straight parallel folds along veins and flowers with a plane unfolded fully were investigated from mechanical point of view by using numerical simulation. Almost regularly parallel veins are observed in leaves of hornbeam, etc. and the veins are angled at $30^{\circ}$ to $50^{\circ}$ from the midrib. This offers the relatively large leaf area in the early stage of unfolding and less compact folded posture in a bud. The extension speed of leaf midrib is very low at an initial stage of unfolding and then it becomes almost constant. This manner corresponds to the unfolding manner of leaves with the lowest energy. The shape of a potato flower fully unfolded looks almost plane. The petal number of a potato flower is usually five or six. This makes the kinetic energy during unfolding smaller and the bad volume larger. From these things, it can be said that the energy during unfolding is more important for plants than the compact posture in a bud.

\section{References}

[1] H. Kobayashi, B. Kresling and J.F.V. Vincent: Proc. Roy. Soc. Lond. Ser. B, Vol. 265 (1998), p. 147.

[2] H. Kobayashi, M. Daimaruya and J.F.V. Vincent: JSME Int. J. Ser. C, Vol. 42 (1999), p. 759.

[3] H. Kobayashi, M. Daimaruya and J.F.V. Vincent: Solid Mech. and Its Appl., Vol. 80 (2000), p.211.

[4] H. Kobayashi, M. Daimaruya and H. Fujita: Solid Mech. and Its Appl., Vol. 106 (2003), p.207.

[5] S. Kaihara and A. Takimoto: Plant and cell Physiol., Vol. 22 (1981), p. 307. 
[6] O. Tanaka, H. Murakami and H. Wada: Bio. Mag.. Tokyo, Vol. 102, (1989), p. 245.

[7] R. U. Larsen and C. Hiden: Scientia Horticulturae, Vol. 63 (1995), p. 225.

[8] R. Bieleski, J. Elgar and J. Heyes: Annals of Botany, Vol. 86 (2000), p. 1175.

[9] B. Kresling: Solid Mech. and Its Applications, Vol. 80 (2000), p.229.

[10] J. M. Delarue: Proc. 3nd Int. Sympo. SFB 230, Stuttgart, (1994) p. 25.

[11] Z. You and S. Pellegrino: AIAA J., Vol. 34 (1996), p. 813.

[12] K. Miura and M. Natori: Space Solar Power Rev., Vol. 5 (1985), p. 345.

[13] S. D. Guest and S. Pellegrino: Proc. 1st Int. Semi. Struct. Morphol., (1992), p. 203.

[14] H. S. Patil and S. Vaijapurkar: J. Bionic Eng., Vol. 4 (2007), p. 19.

[15] A. Herbig and U. Kull: Proc. 2nd Int. Sympo. SFB 230 Part 2, Stuttgart, (1991), p. 109.

[16] R. Yamamoto, Growth of Plant Cells (Baifukan, Japan 1999), p. 47 (in Japanese). 